\title{
B cell reductive therapy with rituximab in the treatment of rheumatoid arthritis
}

\author{
This article was published in the following Dove Press journal: \\ Biologics: Targets \& Therapy \\ II May 2009 \\ Number of times this article has been viewed
}

\section{Joseph M Tuscano',2 \\ Jacob Sands ${ }^{1,2}$}

'Hematology and Oncology, Veterans

Affairs, Northern California

Health Care System, California,

USA; 'Division of Hematology

and Oncology, University of California,

Davis School of Medicine, Sacramento,

California, USA
Correspondence: Joseph M Tuscano Department of Internal Medicine, University of California, Davis Cancer Center, Sacramento, CA 95817, USA $\mathrm{Tel}+|916734377|$

$\mathrm{Fax}+$ I 9167347946

Email joseph.tuscano@ucdmc.ucdavis.edu
Abstract: The approach to treating autoimmune disorders is currently undergoing a significant change in focus. As therapies are developed that are more precise in targeting the pathogenesis for these diseases, patients experience significantly fewer side effects. At the same time, as more precise therapies are discovered, the etiologies of these diseases become further elucidated. It is now widely accepted that B-lymphocytes play a significant role in the pathogenesis of various autoimmune diseases, the extent of which continues to be the focus of ongoing research. Rheumatoid arthritis is one such disease process that has been the focus of various B-lymphocytedirected therapeutic trials. In this paper we review the current research available on rituximab as treatment for rheumatoid arthritis. This review details results from four main studies, as well as others, which used rituximab in at least one of the arms in treatment of rheumatoid arthritis. The results are promising and will likely lead to longer term studies as well as a potential focus on B cell subsets.

Keywords: rheumatoid arthritis, rituximab, treatment efficacy, autoimmune, B-cells

\section{Introduction}

Treatment for autoimmune disorders is in the midst of a significant change. Therapies with nonspecific immunosuppressive agents are being replaced with therapies that have specific targets that have selective effects on a wide range of cell types, cytokines, and signaling pathways. Targeted therapies for autoimmune disease will not only provide the promise of improved efficacy and decreased toxicity, but will also deliver new insights into disease pathogenesis.

Rheumatoid arthritis (RA) is a relatively common disorder, affecting about $1 \%$ of the population, both in the United States and throughout the world. ${ }^{1}$ The etiology is not completely understood, but some important details have recently been elucidated. As the disease becomes better understood, specific targets are being identified opening the door to an array of therapeutic options. Rituximab, a chimeric monoclonal antibody (mAb) that targets human CD20, was approved by the US Food and Drug Administration (FDA) in 2006 for the treatment of RA and is the subject of this review.

RA results from the proliferation of synovial tissue, increased production of synovial fluid, and eventually destruction of bone and cartilage. Although RA was previously believed to be a disease affecting only the joints, it has now been shown to have significant systemic affects, with associated morbidity and mortality. The role of humoral versus cellular immune activity in the resultant disease process is not completely known although recent data suggests both are involved. T-cells are 
activated by an unknown initiating process resulting in the production of interleukin-1 (IL-1) and tumor necrosis factor$\alpha(\mathrm{TNF}-\alpha)$, which have been shown to have a significant role in the inflammatory process. It is believed that after initiation; autoantigens develop, perpetuating the T-cell activity and the disease process. Based on this, RA was previously thought of as a disease mediated primarily by T-cells. This led to a number of clinical trials that utilized mAbs to reduce $\mathrm{T}$ cell numbers, including $\mathrm{T}$ cell subsets. One such trial used an anti-CD4 mAb to reduce the numbers of CD4 + T cells. However, this and other $\mathrm{T}$ cell reductive trials failed to produce any significant clinical benefit suggesting that $\mathrm{T}$-cells are not the sole pathologic focus of the disease process. ${ }^{2}$ More recently, the role of B-cells has been explored both in vitro and in vivo and have been found to play a significant role in the pathogenesis of this disease. Based on this data there has been increasing interest in the use of B cell reductive therapy, with agents like rituximab for the treatment of RA. ${ }^{3}$

The primary mechanism by which B cells are thought to mediate the RA pathologic process is by autoantibody production. However B cells are also capable of antigen presentation to $\mathrm{T}$ cells for expansion of the immune response. While the antigen that is presented to activate T-cells in RA is not known, B cells are thought to mediate the destructive process. B cells also activate macrophages and dendritic cells, promoting the inflammatory process that occurs in the synovia of patients with RA. ${ }^{4} \mathrm{~B}$ cells mature into plasma cells, which produce rheumatoid factor (RF), present in approximately $80 \%$ of patients with RA. RF is an antibody that binds to the Fc region of immunoglobulin $\mathrm{G}(\mathrm{IgG})$ and generally portends a poorer prognosis. Interestingly, RF is also sometimes found in people without RA, but this RF is IgM and often transient with low affinity for macrophages and neutrophils. Conversely, in RA, RF is a high affinity IgG with the capability of migration into extravascular spaces. ${ }^{5}$ Due to the low specificity of RF, anti-cyclic citrullinated peptide antibody (CCP) is also used in diagnosis of rheumatoid arthritis given the high specificity of this antibody as well as increased sensitivity when using both tests. AntiCCP may be especially helpful in early diagnosis of RA. ${ }^{6,7}$ In one meta-analysis assessing IgM RF and anti-CCP, the sensitivity and specificity of $\operatorname{IgM}$ RF was $69 \%$ and $85 \%$ respectively, compared with $67 \%$ and $95 \%$ for anti-CCP. ${ }^{8}$ The authors recommended anti-CCP be used alone in testing patients with low pretest probability for RA to prevent excessive false positives. They recommended testing both $\mathrm{RF}$ and anti-CCP in patients with higher pretest probability to increase sensitivity, allowing for early treatment.
As the etiology of autoimmune diseases becomes better understood, more targeted therapeutics can be developed. These targeted approaches will begin to dissect the immune response in vivo and give us a better understanding of the pathogenic processes in patients with RA. While recent studies utilize rituximab as B cell reductive therapy generate impressive responses they also teach us that the B cell likely has a role beyond production of autoantibodies, as evidenced by the improvement in the clinical picture of patients with RA despite stable autoantibody levels. ${ }^{9,10}$

\section{The role of $B$ cells in the pathogenesis of RA Autoantibodies}

The mere presence of autoantibodies is prima facie evidence for a role for B cells in autoimmune disease pathogenesis. Autoantibody levels are incorporated into diagnostic and prognostic criteria for clinical assessment, and serve as surrogate markers of disease activity. For example, the presence of anti-nuclear antibodies (ANA) serves as a very sensitive diagnostic marker for systemic lupus erythematosis (SLE). ${ }^{11}$ However, this serologic marker is observed in a variety of other autoimmune disorders, and the autoantibody level does not correlate with the severity of disease. However, many autoantibody levels poorly correlate with disease severity and are not thought to play a role in disease pathogenesis. This may only reflect our lack of understanding of disease pathogenesis, as there are many other autoantibodies that provide highly specific criteria for diagnosis of a clinical syndrome. Besides the rheumatologic disorders, examples of pathologic autoantibodies include the antiacetylcholine receptor $(\mathrm{AChR})^{12}$ and the anti-glomerular basement membrane (GBM) antibodies in myasthenia gravis and Goodpastures syndrome, respectively. ${ }^{12,13}$ The beneficial effect of suppression and removal of these pathologic antibodies with immunosuppressive agents or plasmapheresis support a definitive role for B cells in the pathogenesis of autoimmune disease.

\section{Immune complex formation}

Autoantibodies produce pathologic effects through various mechanisms including binding to antigens, immune complex (IC) formation, tissue deposition, complement and Fc receptor activation. Immune complexes are seen in various autoimmune diseases and the effects can be diffuse, including vasculitis, pulmonary hemorrhage, and renal failure. Essential mixed cryoglobulinemia is an example of an IC-mediated disease. Deposition of immune complexes may result in 
cutaneous purpuric lesions, mononeuritis multiplex, and glomerulonephritis. ${ }^{12}$ Immunosuppressive drugs, or even plasmapheresis, are given to reduce immune complex formation by decreasing cryoglobulin production.

In some studies, genetic differences in Fc receptors have been associated with various autoimmune diseases, such as immune thrombocytopenia (ITP), SLE, and multiple sclerosis (MS). ${ }^{14,15}$ It is suspected that the different Fc receptors may result in longer IC circulation, resulting in deposition in tissue, inflammatory reactions, and proliferative responses of FcR-bearing cells. Ultimately tissue destruction is observed.

The presence of ICs also results in additional indirect effects such as complement activation, production of proinflammatory compounds, increased phagocytosis, and enhanced cellular and humoral immune responses, which also produce autoimmune disease. ${ }^{16}$

\section{Antibody-independent B cell dependent mechanisms}

Although B cells produce antibodies, there are other mechanisms by which they cause autoimmune disease, likely through effects on $\mathrm{T}$ cell activity. MLR/lpr autoimmune prone mice engineered not produce immunoglobulin, but with otherwise normal B cell functions still develop interstitial nephritis, vasculitis, and glomerulonephritis. Mortality is also similar to the mice made to retain the ability to secrete immunoglobulin. ${ }^{17}$ Moreover JHD MLR/lpr mice made deficient of B cells develop significantly less disease than their normal B cell producing counterparts. These results suggest $\mathrm{B}$ cells are integral to autoimmune disease pathogenesis by methods other than immunoglobulin production. ${ }^{18}$ This has been hypothesized to be secondary to increased $\mathrm{T}$ cell activation via antigen presentation by B cells.

Intravenous immunoglobulin (IVIG) is used to treat symptoms of ITP, specifically increased platelet destruction by an autoimmune process involving platelet surface glycoproteins. ${ }^{19,20}$ The possible mechanisms of action of IVIG imply complement and Fc receptors (FcR) have a role in B cell mediated autoimmunity. IVIG may have its effect via inhibition of complement or by blocking the FcR, resulting in decreased clearance of platelets coated antibody. There is evidence that in a murine model of ITP, the presence of the $\operatorname{Fc}(\gamma)$ RIIB receptor has a significant impact on effectiveness of IVIG in treating ITP. ${ }^{21}$ Rituximab has also shown a significant response in treating patients with ITP who have failed other therapies, including steroids and splenectomy. ${ }^{22-25}$ suggesting a B cell-mediated pathogenesis.
Response to rituximab in ITP patients is observed as two possible time courses, suggesting different mechanisms of action. There are some that respond within days, potentially through inhibition of FcRs, but others respond over weeks, which may be due to B cell depletion. Though IVIG and rituximab have shown some efficacy, it is limited, suggesting other mechanisms of pathogenesis besides those that are $\mathrm{B}$ cell-mediated, that remain unknown.

\section{Rituximab}

Rituximab is a chimeric human/mouse IgG1 antibody directed at human CD20, which is found on only pre-B and mature B cells. CD20 is not found on plasma cells, stem cells, or pro-B cells, and therefore eliminating the CD20 positive population does not prevent recovery of mature B cells or immunoglobulin production. Rituximab has been used and was initially FDA approved for the treatment of B cell lymphoma in 1997. More than $90 \%$ of B-cell non-Hodgkin's lymphoma (NHL) express CD20,${ }^{26}$ making a CD20 an ideal therapeutic target. Various trials have been completed to evaluate the efficacy of rituximab both in combination and as a single agent. Many trials have shown rituximab to have significant efficacy as therapy for various subtypes of NHL and it is now the established standard of care for most subtypes of B cell NHL. ${ }^{27}$ Rituximab is very well tolerated with grade 1-2 infusion reactions as the most common adverse event, but this was typically observed after only the first dose and has rarely limited administration.

\section{Rituximab for the treatment of RA}

As for the treatment of NHL the optimal dose and schedule of rituximab for the treatment of RA has not been established. However several phase II trials have demonstrated clinical efficacy with a range of doses from $375 \mathrm{mg} / \mathrm{m}^{2}$ weekly for four weeks, $500 \mathrm{mg}$ given as two doses or a single dose of $1000 \mathrm{mg}$. All have been well tolerated with a mean terminal half-life of 19-22 days after the second infusion but a systemic clearance time of about 240 days and a volume of distribution of about 3.6-4.5 L. The pharmacokinetics of rituximab has been shown to be unchanged when used in combination with cyclophosphamide (CTX) or methotrexate (MTX), both of which have demonstrated efficacy when used in combination with rituximab in patients with RA. ${ }^{29,30}$ Rituximab has been shown to be well tolerated without significant side effects ${ }^{29}$ in patients with RA. There have been some pharmacokinetic differences between the dosing for non-Hodgkin's lymphoma and RA, although the clinical implications of this difference are not known. Though it is 
dosed by body surface area for non-Hodgkin's lymphoma, standardized rituximab dosing for rheumatoid arthritis works well for a broad range of body surface areas. ${ }^{30}$ This is possibly due to the enormous variation in target density in patients with NHL.

There have been several randomized clinical trials that have demonstrated clinical efficacy in RA patients that have failed standard treatment approaches (Table 1). The DANCER trial is a randomized, placebo-controlled trial that examined two different doses of rituximab in patients with active moderate to severe RA that have failed previous treatments with disease-modifying antirheumatic drugs (DMARDs) (beyond MTX) as well as biologic response modifiers. ${ }^{31}$ Patients in this study were either previously treated with MTX or were being treated throughout the study, however they were not taking any other DMARD or other biologic therapy during the study period. Patients were randomized to three different treatment arms; (all containing MTX), placebo (MTX alone) versus rituximab $500 \mathrm{mg} \times 2$, versus rituximab $1000 \mathrm{mg} \times 2$. At the end of the study (24-weeks), the response rate was significantly better in each of the rituximab groups when compared to MTX alone. The $500 \mathrm{mg}$ and $1000 \mathrm{mg}$ doses show little differences in the ACR20 and 50 groups, but of those achieving an ACR70 response, the $1000 \mathrm{mg}$ group had a more significant response.

In patients that received steroid treatment or pre-treatment there was no statistical difference in efficacy, suggesting methylprednisolone and prednisone do not affect treatment outcomes in patients receiving rituximab. However, there was a difference in infusion-related side effects suggesting that premedication with steroids can reduce the incidence and severity of rituximab-mediated infusion reactions (which were noted more frequently with the first infusion).

In addition to clinical endpoints, biologic measures also improved in the rituximab treatment arms, which included a significant decrease in C-reactive protein (CRP) levels and RF titers. The vast majority of reported adverse events in the treatment groups were mild to moderate in severity and were similar in all treatment groups with the exception of infusion-related toxicity, which was higher in the treatment arms that included rituximab.

The DANCER study demonstrated significant clinical benefit when rituximab was used in combination with MTX in RF-positive patients who have failed previous treatment with DMARDs. In this study there was no significant difference between the $500 \mathrm{mg}$ and $1000 \mathrm{mg}$ rituximab treatment groups. The study results also suggest a benefit for the use of methylprednisolone pre-medication for the prevention of infusion-related toxicity. Other toxicities were modest and balanced among the treatment groups. In terms of immunosupression there was no significant change in overall immunoglobulin levels and anti-tetanus titers.

In addition, a recent report of the same study assessed healthrelated quality of life which revealed statistically significant improvements in the $500 \mathrm{mg}$ and $1000 \mathrm{mg}$ rituximab-treated groups compared to MTX alone for physical function, pain,

Table I The proportion of patients with American College of Rheumatology criteria scores (ACR) at 24 weeks. Rituximab dosing was $1000 \mathrm{mg}$ with the noted exception

\begin{tabular}{|c|c|c|c|c|c|c|}
\hline Primary author & Treatment & Patients (n) & ACR20 \% (p) & ACR50 \% (p) & ACR70 \% (p) & $\begin{array}{l}\text { Serious adverse } \\
\text { event (\%) }\end{array}$ \\
\hline \multirow[t]{3}{*}{ Emery (DANCER) } & MTX + placebo rituximab & 149 & 28 & 13 & 5 & 18 \\
\hline & Rituximab $500 \mathrm{mg}+\mathrm{MTX}$ & 124 & $55(<0.001)$ & $33(<0.001)$ & $13(0.029)$ & 17 \\
\hline & Rituximab + MTX & 192 & $54(<0.001)$ & $34(<0.001)$ & $20(<0.00 I)$ & 17 \\
\hline \multirow[t]{2}{*}{ Cohen (REFLEX) } & MTX + placebo rituximab & 201 & 18 & 5 & I & 20 \\
\hline & Rituximab + MTX & 298 & $5 \mathrm{I}(<0.000 \mathrm{I})$ & $27(<0.000 I)$ & $12(<0.000 I)$ & 18 \\
\hline \multirow[t]{4}{*}{ Strand } & MTX + placebo rituximab & 40 & 15 & 5 & 2 & \\
\hline & Rituximab & 40 & $26(<0.05)$ & 13 & 6 & \\
\hline & Rituximab + MTX & 40 & $29(<0.01)$ & $17(<0.01)$ & $9(<0.05)$ & \\
\hline & Rituximab + CTX & 41 & $31(<0.0 I)$ & $17(<0.01)$ & 6 & \\
\hline \multirow[t]{4}{*}{ Edwards } & MTX + placebo Rituximab + CTX & 40 & 38 & 13 & 5 & 8 \\
\hline & Rituximab + placebo MTX \& CTX & 40 & $65(0.025)$ & $33(0.059)$ & 15 & 5 \\
\hline & Rituximab + MTX + placebo CTX & 40 & $73(0.003)$ & $43(0.005)$ & $23(0.048)$ & 8 \\
\hline & Rituximab + CTX + placebo MTX & 41 & $76(0.001)$ & $4 I(0.005)$ & 15 & 15 \\
\hline
\end{tabular}

Abbreviations: CTX, cyclophosphamide; MTX, methotrexate. 
vitality, social function, and role-physical subscale scores. ${ }^{32}$ This study also demonstrated a significant improvement in Health Assessment Questionnaire results with the $1000 \mathrm{mg}$ rituximab group compared to MTX alone by week 8 . The $500 \mathrm{mg}$ rituximab group first demonstrated significant improvement at week 12 and these were maintained over the 24 weeks of the assessment period. Both rituximab groups also demonstrated significant improvements in fatigue scores at 12 week, which persisted to the end of the study. However there was no significant difference in the mental component summary. The authors point out that effects are likely seen in pain, mobility, physical function and physical role activity scales before being seen in emotional well-being scales, which has been observed in other studies that utilize biologics including; etanercept, ${ }^{33,34}$ adalimumab, ${ }^{35,36}$ and infliximab. ${ }^{34,37}$

Another large randomized trial included participants that have been heavily pretreated with multiple agents including DMARDs and biologics, (REFLEX trial; ${ }^{38}$ Table 1). The participants in this trial had active rheumatoid arthritis despite prior therapy with MTX and anti-TNF agents including infliximab, adalimumab, or etanercept. Patients were randomized to either placebo or rituximab. Both groups continued to take MTX and methylprednisolone prior to and after enrolment. Though this study was designed for two-year follow-up, the initial publication reported an assessment 24 weeks after treatment initiation. At 24 weeks there was a significant improvement in the rituximab group compared to placebo with $51 \%$ and $18 \%$ achieving an ACR20 response respectively. Similar to what was observed in the DANCER trial, significant initial responses were observed as early as 8 weeks after treatment initiation. ${ }^{32}$ The ACR50 and 70 responses were also significantly improved in the rituximab group relative to placebo starting at weeks 12 and 16 . CRP and erythrocyte sedimentation rate (ESR) levels were improved in the rituximab treatment arms as well. Radiographic changes were compared and demonstrated a trend toward less progression of joint damage in rituximab-treated patients. Significant reduction of joint narrowing scores was observed in the rituximab treated patients compared to placebo as measured by the Genant-modified Sharp assessment. CD19 + cells were assessed to evaluate peripheral $B$ cell levels which revealed a rapid and significant decrease after rituximab dosing, with some recovery of B cell counts beginning between weeks 16 and 20, however mean immunoglobulin levels showed no significant change. The adverse events reported were similar between groups, and like the DANCER trial, many were mild or moderate. There was no significant difference in the incidence of infections between the two groups.

In summary, similar to the results of the DANCER trial, significant improvements were noted in many of the measured outcomes in those patients receiving rituximab and MTX when compared with MTX alone. The REFLEX trial demonstrated that heavily pretreated patients treated with rituximab had significant improvements in nearly all areas that were assessed including: swollen and tender joint counts, patient's and physician's global assessments of disease activity, pain scores, HAQ DI scores, CRP and ESR levels, mental and physical health scores (measured by SF-36), fatigue scores, and a trend toward improvement in joint narrowing assessed radiogaphically.

Two additional smaller, placebo-controlled, double-blind studies that assessed the use of rituximab for the treatment of RA also deserve mention. These studies also included treatment groups that received rituximab alone, MTX alone as well as rituximab plus CTX. ${ }^{10,39}$ ACR20, 50, and 70 scores improved in the rituximab treatment groups when compared to MTX alone (Table 1). Edwards and colleagues continued to follow the various treatment arms to week $48 .{ }^{10}$ ACR20 and 50 criteria continued to demonstrate significant improvements in the patients treated with rituximab and MTX, as well as patients treated with rituximab and CTX. Edwards and colleagues also measured CD-19 + B cell levels and similar to the previously mentioned studies, they also demonstrated a significant reduction post-rituximab treatment without significant change in immunoglobulin levels. RF levels decreased initially in all treatment arms, but the rituximab treatment arms were more likely to be sustained at week 12 and continued to be lower at week 24 compared to MTX alone. Like the DANCER and REFLEX trials, the incidence of infection was not significantly different between the different treatment arms. The incidence of adverse events was similar in all treatment groups. Additionally, $85 \%-90 \%$ of adverse events that occurred with rituximab infusions were mild to moderate in severity and again were more likely after the first infusion.

In a study conducted by Strand and colleagues, the treatment groups were followed for two years..$^{39}$ At 104 weeks, patients treated with rituximab and MTX demonstrated persistent clinical improvement when compared to other treatment groups. Although the rituximab and MTX group showed higher numbers of patients achieving ACR20, 50 , and 70 scores when compared to all other treatment groups at 104 weeks, this was not statistically significant. However assessments via Health Assessment Questionnaire 
measurements demonstrated significant improvements at week 72 compared to baseline in all treatment groups including MTX alone, although the magnitude of the improvement was greater in the treatment groups that included rituximab.

More recently, analysis of the synovia of patients with RA receiving rituximab has demonstrated reduction of $B$ cells and inhibition of structural joint damage. ${ }^{40-43}$ Persistence of RA symptoms despite treatment with rituximab has been shown to correlate with persistence of both plasma cells as well as synovial B cells. ${ }^{41}$ Thurlings and colleagues hypothesize that rituximab has "an indirect effect on short-lived autoreactive plasma cells that are associated with the production of autoantibodies".

As the efficacy of rituximab has become more established, there are studies directed at understanding which patients will best respond to rituximab treatment for RA. Antibody analysis of synovia has shown clinical improvement related to reduction of $\operatorname{IgG}, \operatorname{IgM}$, and $\operatorname{Ig\kappa } .{ }^{42}$ Interestingly, reduction of interleukin-8 was observed in clinical nonresponders. Peripheral IgD + CD27 + memory B cell levels at the time of B cell recovery have also been shown to be significantly higher in nonresponders as well as early relapse. ${ }^{44}$ Initial studies had shown no significant difference in peripheral $\mathrm{B}$ cell numbers between responders to rituximab and nonresponders. Dass and colleagues performed an analysis of patients receiving rituximab using a highly sensitive technique for detecting B cells. ${ }^{45}$ Utilizing MRD flow cytometry techniques, B cells were measured at lower numbers than conventional analysis is able to detect. The results demonstrated a statistically significant decrease in peripheral B cell numbers amongst responders relative to nonresponders. Those who had undetectable peripheral B cell levels after the first infusion were more likely to have a better clinical response as well. Interestingly, those who had a complete B cell depletion after the second infusion, still were less likely to show significant clinical improvement relative to those with depletion after the first infusion, making the rate of peripheral B cell depletion a seemingly important reason for, or surrogate to, clinical improvement.

This observation is further supported by a small study conducted by Thurlings and colleagues, which analyzed the effects of rituximab after three doses. ${ }^{46}$ Patients that did not respond after the first dose showed no significant reduction in symptoms after future doses, suggesting a predictable response to effectiveness of rituximab after the first dose. Repeat dose timing was based upon symptom relapse. These results further strengthen the understanding that rituximab has effectiveness because reduction of the number of B cells results in decreased autoantibody production and therefore less structural joint damage, which then creates improved clinical symptoms. Inhibiting this process clearly has efficacy. It continues to remain unknown if those that do not respond are affected by another process or if they would respond to higher doses.

\section{Conclusion}

Rheumatoid arthritis is a destructive and debilitating disease that affects millions of adults and children around the world. While there are common and typical clinical features, there is significant heterogeneity in the details and severity of the clinical phenotype. This heterogeneity likely reflects considerable variation in the pathogenesis and reaction to the autoimmune process in a given patient. Nonspecific anti-inflammatory agents have provided considerable relief for patients for many years, although typically limited and often at the cost of considerable toxicity. Newer targeted approaches to this disease are providing more effective and less toxic treatment approaches. More importantly, as we further dissect the immune response and develop more specifically targeted agents, these provide not only the potential for improved clinical outcome but also tools for discovery that will allow for a better understanding of the pathogenesis of this disease. Clearly there are multiple components of the immune system that are involved in the pathogenesis of RA, but the studies described herein confirm that B cells play an important role, and therapeutics that specifically provide B cell reduction have become an accepted weapon in the arsenal against this dreadful disease. It is important to note that the studies done thus far had a short follow-up when considering a potential lifetime therapy with rituximab. The side effect profile, as well as efficacy, may change when treating for many years. Future studies will focus on the long-term effects of B cell reductive therapy, ideal schedule for rituximab dosing, and the efficacy of targeting specific B cell subsets.

\section{Disclosure}

The authors report no conflicts of interest in this work.

\section{References}

1. O'Dell JR. Cecil Medicine. 23rd ed. Philadelphia, PA: Saunders; 2007.

2. van der Lubbe PA, Breedveld FC, Tak PP, Schantz A, Woody J, Miltenburg AM. Treatment with a chimeric CD4 monoclonal antibody is associated with a relative loss of CD $4+/ \mathrm{CD} 45 \mathrm{RA}+$ cells in patients with rheumatoid arthritis. J Autoimmun. 1997;10(1):87-97.

3. Tanaka Y. Treatment of inflammatory immunologic disease 4. B cell targeting therapy using the anti-CD20 antibody rituximab in inflammatory autoimmune diseases. Intern Med. 2007;46(16):1313-1315. 
4. Bayry J, Lacroix-Desmazes S, Kazatchkine MD, Kaveri SV Monoclonal antibody and intravenous immunoglobulin therapy for rheumatic diseases: rationale and mechanisms of action. Nat Clin Pract Rheumatol. 2007;3(5):262-272.

5. Thompson KM, Borretzen M, Randen I, Forre O, Natvig JB. V-gene repertoire and hypermutation of rheumatoid factors produced in rheumatoid synovial inflammation and immunized healthy donors. Ann N Y Acad Sci. 1995;764:440-449.

6. Rantapaa-Dahlqvist S, de Jong BA, Berglin E, et al. Antibodies against cyclic citrullinated peptide and $\operatorname{IgA}$ rheumatoid factor predict the development of rheumatoid arthritis. Arthritis Rheum. 2003;48: 2741-2749.

7. Berglin E, Padyukov L, Sundin U, et al. A combination of autoantibodies to cyclic citrullinated peptide (CCP) and HLA-DRB1 locus antigens is strongly associated with future onset of rheumatoid arthritis. Arthritis Res Ther. 2004;6:R303-R308.

8. Nishimura K, Sugiyama D, Kogata Y, et al. Meta-analysis: Diagnostic accuracy of anti-cyclic cintrullinated peptide antibody and rheumatoid factor for rheumatoid arthritis. Ann Intern Med. 2007;146: 797-808.

9. Higashida J, Wun T, Schmidt S, Naguwa SM, Tuscano JM. Safety and efficacy of rituximab in patients with rheumatoid arthritis refractory to disease modifying antirheumatic drugs and anti-tumor necrosis factoralpha treatment. J Rheumatol. 2005;32(11):2109-2115.

10. Edwards JC, Szczepanski L, Szechinski J, et al. Efficacy of B-celltargeted therapy with rituximab in patients with rheumatoid arthritis. N Engl J Med. 2004;350(25):2572-2581.

11. Davidson A DB. Autoimmune diseases. N Engl J Med. 2001;345(5): 340-350.

12. Hudson BG, Tryggvason K, Sundaramoorthy M, Neilson EG. Alport's syndrome, Goodpasture's syndrome, and type IV collagen. $N$ Engl J Med. 2003;348(25):2543-2556.

13. Richman DP, Agius MA. Treatment of autoimmune myasthenia gravis. Neurology. 2003;61(12):1652-1661.

14. Ravetch JV, Bolland S. IgG Fc receptors. Annu Rev Immunol. 2001;19:275-290.

15. Binstadt BA, Geha RS, Bonilla FA. IgG Fc receptor polymorphisms in human disease: implications for intravenous immunoglobulin therapy. J Allergy Clin Immunol. 2003;111(4):697-703.

16. Holers VM. The complement system as a therapeutic target in autoimmunity. Clin Immunol. 2003;107(3):140-151.

17. Chan OT, Hannum LG, Haberman AM, Madaio MP, Shlomchik MJ. A novel mouse with B cells but lacking serum antibody reveals an antibody-independent role for B cells in murine lupus. J Exp Med. 1999;189(10):1639-1648.

18. Shlomchik MJ, Madaio MP, Ni D, Trounstein M, Huszar D. The role of B cells in 1pr/lpr-induced autoimmunity. J Exp Med. 1994;180(4): 1295-1306.

19. McMillan R. Antiplatelet antibodies in chronic adult immune thrombocytopenic purpura: assays and epitopes. J Pediatr Hematol Oncol. 2003;25(Suppl 1):S57-S61.

20. McMillan R, Lopez-Dee J, Loftus JC. Autoantibodies to alpha (IIb)beta(3) in patients with chronic immune thrombocytopenic purpura bind primarily to epitopes on alpha(IIb). Blood. 2001;97(7): 2171-2172.

21. Samuelsson A, Towers TL, Ravetch JV. Anti-inflammatory activity of IVIG mediated through the inhibitory Fc receptor. Science. 2001; 291(5503):484-486.

22. Stasi R, Pagano A, Stipa E, Amadori S. Rituximab chimeric anti-CD20 monoclonal antibody treatment for adults with chronic idiopathic thrombocytopenic purpura. Blood. 2001;98(4):952-957.

23. Saleh MN, Gutheil J, Moore M, et al. A pilot study of the anti-CD20 monoclonal antibody rituximab in patients with refractory immune thrombocytopenia. Semin Oncol. 2000;27(6 Suppl 12):99-103.

24. Zaja F, Vianelli N, Sperotto A, et al. B-cell compartment as the selective target for the treatment of immune thrombocytopenias. Haematologica. 2003;88(5):538-546.
25. Cooper N, Stasi R, Cunningham-Rundles S, et al. The efficacy and safety of B-cell depletion with anti-CD20 monoclonal antibody in adults with chronic immune thrombocytopenic purpura. Br J Haematol. 2004;125(2):232-239.

26. Riley JK, Sliwkowski MX. CD20: a gene in search of a function. Semin Oncol. 2000;27(6 Suppl 12):17-24.

27. McLaughlin P, Grillo-Lopez AJ, Link BK, et al. Rituximab chimeric anti-CD20 monoclonal antibody therapy for relapsed indolent lymphoma: half of patients respond to a four-dose treatment program. J Clin Oncol. 1998;16(8):2825-2833.

28. Coiffier B, Lepage E, Briere J, et al. CHOP chemotherapy plus rituximab compared with CHOP alone in elderly patients with diffuse large-B-cell lymphoma. N Engl J Med. 2002;346(4):235-242.

29. Breedveld F, Agarwal S, Yin M, et al. Rituximab pharmacokinetics in patients with rheumatoid arthritis: B-cell levels do not correlate with clinical response. J Clin Pharmacol. 2007;47(9):1119-1128.

30. Ng CM, Bruno R, Combs D, Davies B. Population pharmacokinetics of rituximab (anti-CD20 monoclonal antibody) in rheumatoid arthritis patients during a phase II clinical trial. J Clin Pharmacol. 2005;45(7):792-801.

31. Emery P, Fleischmann R, Filipowicz-Sosnowska A, et al. The efficacy and safety of rituximab in patients with active rheumatoid arthritis despite methotrexate treatment: results of a phase IIB randomized, double-blind, placebo-controlled, dose-ranging trial. Arthritis Rheum. 2006;54(5):1390-1400.

32. Mease PJ, Revicki DA, Szechinski J, et al. Improved health-related quality of life for patients with active rheumatoid arthritis receiving rituximab: Results of the Dose-Ranging Assessment: International Clinical Evaluation of Rituximab in Rheumatoid Arthritis (DANCER) Trial. J Rheumatol. 2008;35(1):20-30.

33. Mathias SD, Colwell HH, Miller DP, Moreland LW, Buatti M, Wanke L. Health-related quality of life and functional status of patients with rheumatoid arthritis randomly assigned to receive etanercept or placebo. Clin Ther. 2000;22(1):128-139.

34. Heiberg MS, Nordvag BY, Mikkelsen K, et al. The comparative effectiveness of tumor necrosis factor-blocking agents in patients with rheumatoid arthritis and patients with ankylosing spondylitis: a sixmonth, longitudinal, observational, multicenter study. Arthritis Rheum. 2005;52(8):2506-2512.

35. Keystone EC, Kavanaugh AF, Sharp JT, et al. Radiographic, clinical, and functional outcomes of treatment with adalimumab (a human anti-tumor necrosis factor monoclonal antibody) in patients with active rheumatoid arthritis receiving concomitant methotrexate therapy: a randomized, placebo-controlled, 52-week trial. Arthritis Rheum. 2004;50(5):1400-1411.

36. Kaplan RM, Groessl EJ, Sengupta N, Sieber WJ, Ganiats TG. Comparison of measured utility scores and imputed scores from the SF-36 in patients with rheumatoid arthritis. Med Care. 2005;43(1): 79-87.

37. Maini RN, Breedveld FC, Kalden JR, et al. Sustained improvement over two years in physical function, structural damage, and signs and symptoms among patients with rheumatoid arthritis treated with infliximab and methotrexate. Arthritis Rheum. 2004;50(4): 1051-1065.

38. Cohen SB, Emery P, Greenwald MW, et al. Rituximab for rheumatoid arthritis refractory to anti-tumor necrosis factor therapy: Results of a multicenter, randomized, double-blind, placebo-controlled, phase III trial evaluating primary efficacy and safety at twenty-four weeks. Arthritis Rheum. 2006;54(9):2793-2806.

39. Strand V, Balbir-Gurman A, Pavelka K, et al. Sustained benefit in rheumatoid arthritis following one course of rituximab: improvements in physical function over 2 years. Rheumatology (Oxford). 2006;45(12):1505-1513.

40. Keystone E, Emery P, Peterfy CG, et al. Rituximab inhibits structural joint damage in patients with rheumatoid arthritis with an inadequate response to tumour necrosis factor inhibitor therapies. Ann Rheum Dis. 2009:68:216-221 
41. Thurlings RM, Vos K, Wijbrandts CA, et al. Synovial tissue response to rituximab: mechanism of action and identification of biomarkers of response. Ann Rheum Dis. 2008;67:917-925.

42. Kavanaugh A, Rosengren S, Lee SJ, et al. Assessment of rituximab's immunomodulatory synovial effects (ARISE trial). 1:clinical and synovial biomarker results. Ann Rheum Dis. 2008;67:402-408.

43. Vos K, Thurlings RM, Wijbrandts CA, et al. Early effects of rituximab on the synovial cell infiltrate in patients with rheumatoid arthritis. Arthritis Rheum. 2007;56(3):772-778.
44. Roll P, Dorner T, Tony H. Anti-CD20 therapy in patients with rheumatoid arthritis. Arthritis Rheum. 2008;58(6):1566-1575.

45. Dass S, Rawstron A, Vital E, et al. Highly sensitive B cell analysis predicts response to rituximab therapy in rheumatoid arthritis. Arthritis Rheum. 2008;58(10):2993-2999.

46. Thurlings RM, Vos K, Gerlag D, et al. Disease activity-guided rituximab therapy in rheumatoid arthritis. Arthritis Rheum. 2008;58(12): 3657-3664.

\section{Publish your work in this journal}

Biologics: Targets \& Therapy is an international, peer-reviewed journal focusing on the patho-physiological rationale for and clinical application of Biologic agents in the management of autoimmune diseases, cancers or other pathologies where a molecular target can be identified. This journal is indexed on PubMed Central, CAS,

\section{Dovepress}

EMBase, Scopus and the Elsevier Bibliographic databases. The manuscript management system is completely online and includes a very quick and fair peer-review system, which is all easy to use. Visit http://www.dovepress.com/testimonials.php to read real quotes from published authors. 